\title{
A one-loop test of quantum supergravity
}

\author{
Sayantani Bhattacharyya ${ }^{a}$, Alba Grassi ${ }^{b}$, Marcos Mariño $^{b}$ and Ashoke Sen ${ }^{a}$ \\ ${ }^{a}$ Harish Chandra Research Institute, \\ Chhatnag Road, Jhusi, Allahabad 211019, India \\ ${ }^{b}$ Département de Physique Théorique et Section de Mathématiques, \\ Université de Genève, Genève, CH-1211 Switzerland \\ sayanta@mri.ernet.in, alba.grassi@unige.ch, marcos.marino@unige.ch, \\ sen@mri.ernet.in
}

\begin{abstract}
The partition function on the three-sphere of ABJM theory and its generalizations has, at large $N$, a universal, subleading logarithmic term. Inspired by the success of one-loop quantum gravity for computing the logarithmic corrections to black hole entropy, we try to reproduce this universal term by a one-loop calculation in Euclidean elevendimensional supergravity on $\mathrm{AdS}_{4} \times X_{7}$. We find perfect agreement between the results of ABJM theory and the eleven dimensional supergravity.
\end{abstract}




\section{Contents}

1. Introduction 1

2. General strategy 3

3. The calculation 5

3.1 Expression for the logarithmic correction 6

3.2 Calculation of the number of zero modes 7

3.3 Calculation of $\beta_{2}$

3.4 Logarithmic correction to the free energy 9

4. Conclusions and outlook 10

\section{Introduction}

Recently, various exact results have been obtained on the partition function on the threesphere of supersymmetric Chern-Simons-matter (CSM) theories generalizing ABJM theory [1]. Typical examples are quiver $\mathcal{N}=3$ CSM theories [2]. These theories are parametrized by the rank $N$ of the $U(N)$ gauge group, the CS levels $k_{a}$, and the number of flavors in each node, $N_{f_{a}}$. Here, $a=1, \cdots, p$ is an index labeling the node. For these theories, the partition function at all orders in $1 / N$ was computed in [3, 迎 by generalizing earlier work in [5, 6, 7, 8]. The result is given by an Airy function

$$
Z_{\mathrm{CFT}}\left(N,\left\{k_{a}\right\},\left\{N_{f_{a}}\right\}\right) \propto \mathrm{Ai}\left[C\left(\left\{k_{a}\right\},\left\{N_{f_{a}}\right\}\right)^{-1 / 3}\left(N-B\left(\left\{k_{a}\right\},\left\{N_{f_{a}}\right\}\right)\right)\right] .
$$

In this expression, $C\left(\left\{k_{a}\right\},\left\{N_{f_{a}}\right\}\right)$ is a known function of the parameters $k_{a}$ and $N_{f_{a}}$. The function $B\left(\left\{k_{a}\right\},\left\{N_{f_{a}}\right\}\right)$ can be computed by a precise algorithm in a case-by-case basis [4]. The proportionality coefficient in (1.1) is independent of $N$, but it is a non-trivial function of $k_{a}$ and $N_{f_{a}}$ (see [4, 9] for explicit results on this coefficient in ABJM theory).

If we recall the asymptotic expansion of the Airy function,

$$
\operatorname{Ai}(x) \sim \frac{\mathrm{e}^{-\frac{2}{3} x^{3 / 2}}}{2 \sqrt{\pi} x^{1 / 4}}, \quad x \gg 1,
$$

we find the large $N$ expansion of the free energy $F \equiv \ln Z$

$$
\begin{aligned}
F_{\mathrm{CFT}}\left(N,\left\{k_{a}\right\},\left\{N_{f_{a}}\right\}\right)= & -\frac{2}{3} C\left(\left\{k_{a}\right\},\left\{N_{f_{a}}\right\}\right)^{-1 / 2} N^{3 / 2}+C\left(\left\{k_{a}\right\},\left\{N_{f_{a}}\right\}\right)^{-1 / 2} B\left(\left\{k_{a}\right\},\left\{N_{f_{a}}\right\}\right) N^{1 / 2} \\
& -\frac{1}{4} \log N+\text { constant }+\mathcal{O}\left(\frac{1}{\sqrt{N}}\right)
\end{aligned}
$$


These CSM theories are conjecturally dual to M-theory compactifications on manifolds of the form $\mathrm{AdS}_{4} \times X_{7}$, where $X_{7}$ is a tri-Sasaki Einstein manifold whose geometry is specified by the data of the quiver $k_{a}, N_{f_{a}}$. In particular, the function $C\left(\left\{k_{a}\right\},\left\{N_{f_{a}}\right\}\right)$ is given by 4, 4, 10]

$$
C\left(\left\{k_{a}\right\},\left\{N_{f_{a}}\right\}\right)=\frac{6 \operatorname{vol}\left(X_{7}\right)}{\pi^{6}},
$$

where in the definition of $\operatorname{vol}\left(X_{7}\right)$ we have taken out a factor of $(2 L)^{7}, L$ being the 'radius' of $\mathrm{AdS}_{4}$. With this convention $\operatorname{vol}\left(X_{7}\right)$ is a purely numerical factor. The leading term of the free energy is then

$$
-\sqrt{\frac{2 \pi^{6}}{27 \operatorname{vol}\left(X_{7}\right)}} N^{3 / 2} .
$$

If we use the dictionary relating $N$ to the AdS radius $L$

$$
\left(2 \pi \ell_{p}\right)^{6} N=6(2 L)^{6} \operatorname{vol}\left(X_{7}\right)
$$

it can be shown that (1.5) is (minus) the regularized, gravitational action on-shell, as expected from the AdS/CFT correspondence (see for example [11] for a review). This provides a non-trivial, quantitative check of the correspondence at leading order in $N$.

The first subleading correction of order $N^{1 / 2} \sim L^{3}$ originates from a local 8-derivative correction to the effective action that modifies the relation between $N$ and $L$ by shifting $N$ by $-B\left(\left\{k_{a}\right\},\left\{N_{f_{a}}\right\}\right)[12$, 13]. A similar shift in the context of five dimensional black hole entropy has been discussed in [14].

What about the subleading, logarithmic correction appearing in (1.3)? When expressed in terms of the AdS radius, it leads to a term of the form

$$
-\frac{3}{2} \log L
$$

Notice that this term is universal: it is the same for all compactifications, irrespectively of the $X_{7}$ manifold. In fact, there is some evidence that, even for theories with $\mathcal{N}=2$ supersymmetry, the partition function is also an Airy function [15], and therefore one finds the same type of logarithmic correction.

A natural question is: can one test the result of this "microscopic," gauge theory computation of the partition function, in terms of a "macroscopic" computation in AdS gravity?

Recently, a similar question has been answered in the affirmative in a related context. In string theory there are by now many exact formulae for the microscopic entropy of extremal black holes, as a function of the charges. In the limit of large charges, this entropy agrees with the Bekenstein-Hawking entropy (or, more generally, with Wald's entropy). However, there are subleading corrections in the asymptotic expansion of the exact microscopic entropy. These include in particular logarithmic corrections. In [16, 17, 18, 19, 20] it has been shown that these logarithmic corrections can be obtained by a 
one-loop calculation in Euclidean quantum gravity. ${ }^{1}$ The field theory background for this calculation is taken to be the near-horizon geometry of the black hole.

Our problem is very similar, structurally, to the problem of computing extremal black hole entropy. The CFT partition function can be regarded as the "microscopic" result for the partition function. The leading large $N$ result (1.5) is the analogue of the BekensteinHawking or Wald entropy. We then expect the subleading logarithmic correction (1.7) to be reproduced by a one-loop correction in Euclidean quantum gravity on $\mathrm{AdS}_{4} \times X_{7}$, as in [16, 17, 18, 19, 20].

In this note we describe a one-loop calculation in eleven-dimensional supergravity (11d SUGRA) leading to a log correction of the form (1.7). The only contribution to the log corrections in 11d SUGRA comes from the analysis of zero modes. This is due to the fact that in odd dimensions the heat kernel expansion does not contain constant terms; an analogous situation occurs in the analysis of five-dimensional black holes [19]. The only source of zero modes in the $\mathrm{AdS}_{4} \times X_{7}$ background is the two-form anticommuting ghost which appears in the quantization of the SUGRA three-form. Since this does not depend on $X_{7}$, this would explain the universality of the result (1.7). Our goal will be to check that the contribution from the zero modes gives us the same coefficient of $\log L$ that appears in (1.7).

\section{General strategy}

The general strategy for computing the logarithmic term in the one-loop corrections in Euclidean quantum gravity has been explained in [16, 17, 18, 19, 20], building on previous results (see for example [22, 23]).

The contribution of a free field to the free energy $F$ is divided into two parts: the contribution of non-zero modes, and the contribution of zero modes. Let us start with the contribution of non-zero modes. This is given by the logarithm of the one-loop determinant of the kinetic operator $A$, sans the zero modes, and takes the form

$$
\mp \frac{1}{2} \ln \operatorname{det}^{\prime} A=\mp \frac{1}{2} \sum_{n}{ }^{\prime} \ln \kappa_{n}
$$

where the ' denotes sum over non-zero modes, $\kappa_{n}$ are the eigenvalues of the kinetic operator, and the sign $\mp$ corresponds to Grassmann even/odd fields, respectively. Information about the spectrum of an operator $A$ is encoded in its heat kernel operator, defined as

$$
K(\tau)=\mathrm{e}^{-\tau A}=\sum_{n} \mathrm{e}^{-\kappa_{n} \tau}\left|\phi_{n}\right\rangle\left\langle\phi_{n}\right|
$$

where $\left|\phi_{n}\right\rangle$ are the corresponding eigenstates (here, for simplicity, we are assuming that the spectrum is discrete and non-degenerate; the formulae can be easily modified for more

\footnotetext{
${ }^{1}$ A general argument showing why the logarithmic corrections are not affected by higher loop corrections can be found in section 2.5 of [21]. Even though the argument was given in the context of black hole entropy, it holds for the partition function of quantum gravity in any background characterized by a large overall length scale.
} 
general cases). As emphasized in for example section 2 of [24], the heat kernel contains information about both zero and non-zero modes. Let us denote by $n_{A}^{0}$ the number of zero modes of the operator $A$. Then, one has the following equation

$$
-\frac{1}{2} \ln \operatorname{det}^{\prime} A=\frac{1}{2} \int_{\epsilon}^{\infty} \frac{\mathrm{d} \tau}{\tau}\left(\operatorname{Tr} K(\tau)-n_{A}^{0}\right)
$$

where $\epsilon$ is an UV cutoff. On the other hand, the trace of the heat kernel has the following well-known expansion at small $\tau$, called the Seeley-De Witt expansion, ${ }^{2}$

$$
\operatorname{Tr} K(\tau)=\frac{1}{(4 \pi)^{d / 2}} \sum_{n=0}^{\infty} \tau^{n-d / 2} \int \mathrm{d}^{d} x \sqrt{g} a_{n}(x, x) .
$$

As explained in [16, 17, 18, 19, 20], one can extract from the Seeley-De Witt expansion the contribution to $\ln \operatorname{det}^{\prime} A$ proportional to $\log L$. To see this, notice that since the nonzero eigenvalues of a standard Laplace type operator $A$ scale as $L^{-2}$, the heat kernel is a function of

$$
\bar{\tau}=\frac{\tau}{L^{2}}
$$

and we can write

$$
-\frac{1}{2} \ln \operatorname{det}^{\prime} A=\frac{1}{2} \int_{\epsilon / L^{2}}^{\infty} \frac{\mathrm{d} \bar{\tau}}{\bar{\tau}}\left(\sum_{n=0}^{\infty} \frac{1}{(4 \pi)^{d / 2}} \bar{\tau}^{n-d / 2} L^{2 n-d} \int \mathrm{d}^{d} x \sqrt{g} a_{n}(x, x)-n_{A}^{0}\right) .
$$

The logarithmic contribution to $\ln \operatorname{det}^{\prime} A$ comes from the term $n=d / 2$ in (2.6), and we get

$$
-\frac{1}{2} \ln \operatorname{det}^{\prime} A=\left(\frac{1}{(4 \pi)^{d / 2}} \int \mathrm{d}^{d} x \sqrt{g} a_{d / 2}(x, x)-n_{A}^{0}\right) \log L+\cdots,
$$

where $\cdots$ denote non-logarithmic contributions. In odd-dimensional spacetimes, as it will be in our case, the coefficient $a_{d / 2}$ vanishes, ${ }^{3}$ and the only contribution comes from $n_{A}^{0}$. Combining (2.1) and (2.7) we get the net contribution to the free energy from the non-zero modes.

Note that the $\log L$ term comes from the region of integration $\epsilon / L^{2} \ll \bar{\tau} \ll 1$ which, in the original variable, translates to $\epsilon \ll \tau \ll L^{2}$. This is the infrared region and hence is not affected by the details of the ultraviolet cut-off $\epsilon$, which only affects the contribution to the integral from the region $\tau \sim \epsilon$. This is important since eleven dimensional supergravity is known to have ultra-violet divergences [25]. The reader may nevertheless worry about the fact that the UV divergent terms could give contributions which dominate over the logarithmic corrections, e.g. a term involving $a_{n}$ will give a one loop contribution of order $L^{d-2 n}$. Thus for example, the $a_{0}$ term, if non-zero, would have produced a contribution of order $L^{11}$ in $d=11$. To avoid this worry we could consider, instead of the free energy $F$, the quantity $(L \mathrm{~d} / \mathrm{d} L-1)(L \mathrm{~d} / \mathrm{d} L-3) \cdots(L \mathrm{~d} / \mathrm{d} L-11) F$. In this all polynomials in $L$

\footnotetext{
${ }^{2}$ The coefficients $a_{n}$ given here were called $a_{2 n}$ in 20 .

${ }^{3}$ Since $\mathrm{AdS}_{4}$ is a manifold with boundary, there could be half integer powers of $\tau$ in the expansion of $\operatorname{Tr} K(\tau)$ from the boundary (see e.g. [26]). However these are given by integrals of local terms over the boundary of $\mathrm{AdS}_{4}$ and can be cancelled by boundary counterterms.
} 
up to order $L^{11}$ cancel, and the dominant term is proportional to $\log L$, whose coefficient we are calculating. A similar trick was used in [27] for extracting the universal part of entanglement entropy in quantum fleld theories.

Let us now look at the contribution coming from zero modes. As in [16, 17, 18, 19, 20], these arise due to asymptotic symmetries. To understand the dependence on $L$ of this integration, one evaluates the Jacobian from the coefficients of the zero modes, to the parameters labeling the supergroup of asymptotic symmetries. Let us suppose that there is a factor of $L^{ \pm \beta_{A}}$ for each zero mode. Then, the total contribution to the partition function from the zero modes is

$$
L^{ \pm \beta_{A} n_{A}^{0}}
$$

and hence to the free energy is

$$
\pm \beta_{A} n_{A}^{0} \log L .
$$

Notice that our conventions for the Grassmannian case are slightly different from the ones used in [18, 19].

At this point, there is an important remark to be made about the computation of the number of zero modes $n_{A}^{0}$. Often in non-compact spaces the number of zero modes, $n_{A}^{0}$, is infinite. Let us first suppose that our space is compact. If we call $\phi_{\ell}^{(0)}(x)$ the normalized eigenfunctions corresponding to the zero modes, where $\ell=1, \cdots, n_{A}^{0}$, we have the equation

$$
n_{A}^{0}=\sum_{\ell=1}^{n_{A}^{0}} \int \mathrm{d}^{d} x \sqrt{g}\left|\phi_{\ell}^{(0)}(x)\right|^{2} .
$$

In a non-compact space this expression is often divergent, leading to an infinite value of $n_{A}^{0}$. Thus in order to make sense of this equation, one has to find a suitable regularization of this expression. In homogeneous spaces of constant curvature, like Euclidean $\mathrm{AdS}_{4}$ or $\mathrm{AdS}_{2} \times \mathbb{S}^{2}$, the sum

$$
\sum_{\ell}\left|\phi_{\ell}^{(0)}(x)\right|^{2}
$$

is a constant. Thus we can express (2.10) as

$$
n_{A}^{0}=\left(\sum_{\ell}\left|\phi_{\ell}^{(0)}(x)\right|^{2}\right) \int \mathrm{d}^{d} x \sqrt{g} .
$$

Even though the sum over $\ell$ runs over an infinite number of zero modes, $\sum_{\ell}\left|\phi_{\ell}^{(0)}(x)\right|^{2}$ is finite in cases of interest. Thus the divergence comes from the infinite volume of spacetime, and evaluation of (2.10) only involves finding a suitable regularization of the volume of space-time. This will be discussed for $\mathrm{AdS}_{4}$ space in $\S 3.2$.

\section{The calculation}

Our goal now is to perform a "macroscopic" calculation of the $\log L$ correction to the free energy of 11d SUGRA on a background of the form $\mathrm{AdS}_{4} \times X_{7}$, and compare it to the microscopic prediction (1.7) from AdS/CFT. 
The most important simplification in our case is the fact that, since we are in odd dimensions, the contributions coming from the Seeley-De Witt expansion in (2.7) vanish. Therefore, we only have to take into account the zero mode contribution (2.9). We are then led to the question of which fields lead to discrete zero modes in the background we are considering. Fields with zero modes play of course a crucial rôle in the calculations of 16, 17, 18, 19, 20]. There, the background is $\mathrm{AdS}_{2} \times \mathbb{S}^{2}$ or $\mathrm{AdS}_{2} \times\left(\right.$ squashed) $\mathbb{S}^{3}$, and the zero modes arise from the "exceptional" zero modes of one-forms, metric and gravitinoes on $\mathrm{AdS}_{2}$ described in [28].

\subsection{Expression for the logarithmic correction}

In (Euclidean) $\mathrm{AdS}_{4}$, the only bosonic fields which might possibly have discrete zero modes are actually two-forms, as explained in [29] (in general, $N$-forms have discrete zero modes on Euclidean $\mathrm{AdS}_{2 N}$ ). For fermionic fields, it can be shown that neither spinors (of spin $1 / 2$ ) nor gravitinos (of spin 3/2) have zero modes.

Now, there is a source of two-forms in the quantization of 11d SUGRA. This is because the quantization of the SUGRA three-form $C_{M N P}$ needs a generalized ghost field which is a Grassmannian two-form. In general, the quantization of a $p$-form $A_{p}$ requires $p$ generalized ghost fields $A_{p-j}$ which are $p-j$ forms, $j=1, \cdots, p$ [30, 31, 32]. They are Grassmann even if $j$ is even, and Grassmann odd if $j$ is odd. The action for the original $p$-form and the ghost fields, after gauge fixing, is given by

$$
S=\frac{1}{2} \sum_{j=0}^{p}(p-j) !\left(A_{p-j},\left(\Delta_{p-j}\right)^{j+1} A_{p-j}\right)
$$

where $(\cdot, \cdot)$ is the standard inner product of forms induced by the Riemannian metric, and $\Delta_{k}$ is the Hodge-Laplace operator acting on $k$-forms. The one-loop contribution to the free energy of the non-zero modes is then given by

$$
-\frac{1}{2} \sum_{j=0}^{p}(-1)^{j}(j+1) \ln \operatorname{det}\left(\Delta_{p-j}^{\prime}\right)
$$

where the ' indicates that we are removing the zero modes. In an odd-dimensional spacetime, $a_{d / 2}$ vanishes and using (2.7), (3.2) we get the logarithmic contribution to the free energy from the non-zero modes to be

$$
-\sum_{j}(-1)^{j}(j+1) n_{\Delta_{p-j}}^{0} \log L
$$

where $n_{\Delta_{p-j}}^{0}$ is the number of zero modes of the Hodge-Laplace operator $\Delta_{p-j}$. Taking into account the contribution of zero modes given in (2.9), we obtain the general expression

$$
\Delta F=\sum_{j}(-1)^{j}\left(\beta_{p-j}-j-1\right) n_{\Delta_{p-j}}^{0} \log L
$$

for the logarithmic contribution to the free energy of all the physical fields and ghost fields appearing in the quantization of a $p$ form. In our case, $n_{\Delta_{p-j}}^{0}$ is only different from zero when $p=3$ and $j=1$. This gives

$$
\Delta F=-\left(\beta_{2}-2\right) n_{\Delta_{2}}^{0} \log L .
$$


Thus we have to compute $n_{\Delta_{2}}^{0}$ and $\beta_{2}$.

Physically the $-\beta_{2}$ factor in (3.5) is the result of integration over the zero modes of the 2 -form field. Since the 2-form field is a ghost field, one might wonder what it means to integrate over its zero modes. For this we can offer the following interpretation. In theories with gauge invariance, the definition of the path integral involves dividing by the volume of the group of gauge transformations $\operatorname{vol}(\mathcal{G})$. In the usual Faddeev-Popov gauge fixing, this factor is cancelled by the ghost path integral. However, when there are zero modes in the Faddeev-Popov operator, there is only a partial cancellation, and after gauge-fixing the path integral still includes a factor of $1 / \operatorname{vol}(\mathcal{H})$, where $\mathcal{H}$ is the subgroup of gauge transformations generated by zero modes (see [11], section 3.1, for a review of this fact in the context of gauge theories, and [33], section 3.4, for an example in gravity). In our case, since the gauge transformation parameters of the 3-form field are given by a 2-form, the path integral will contain in the denominator an integration over the zero modes of the two-form fields. The contribution in (3.5) proportional to $\beta_{2}$ can then be interpreted as the result of dividing the path integral by the integral over the zero modes of the gauge transformation parameter. This also explains why this contribution comes with a minus sign.

Notice that there is another potential source of zero modes, - these could arise if $X_{7}$ has a harmonic one-form so that we can get a harmonic three-form on $\mathrm{AdS}_{4} \times X_{7}$ by taking the wedge product of a harmonic two-form on $\mathrm{AdS}_{4}$ times a harmonic one-form on $X_{7}$. However, the $X_{7}$ are compact Einstein manifolds of positive curvature, and they have $b_{1}=0$ (see for example [34, page 57). Thus we conclude that there are no zero modes from this decomposition.

\subsection{Calculation of the number of zero modes}

To compute $n_{\Delta_{2}}^{0}$, we use 2.12$)$. Thus we need to calculate two quantities: $\sum_{\ell}\left|\phi_{\ell}^{(0)}(x)\right|^{2}$ and the regularized volume of $\mathrm{AdS}_{4}$. For the first quantity we can use the general result of [29], which says that on $\mathrm{AdS}_{M}$ and for $M / 2$-forms,

$$
\sum_{\ell}\left|\phi_{\ell}^{(0)}(x)\right|^{2}=\frac{1}{2^{M} \pi^{M / 2}} \frac{M !}{(M / 2) !} \frac{1}{L^{4}} .
$$

For $M=4$ this gives

$$
\sum_{\ell}\left|\phi_{\ell}^{(0)}(x)\right|^{2}=\frac{3}{4 \pi^{2} L^{4}} .
$$

We can also arrive at this result by explicitly evaluating the left hand side at the origin of $\mathrm{AdS}_{4}$. In this case only a few $\phi_{\ell}(x)$ 's are non-vanishing, and by explicitly summing over the contribution from these modes we again arrive at (3.7)

The regularized volume of $\mathrm{AdS}_{4}$ can be calculated by standard procedure (see for example [35, 6]) but since this forms an integral part of our analysis, we shall review it here. For this we write the $\mathrm{AdS}_{4}$ metric as

$$
\mathrm{d} s^{2}=L^{2}\left(\mathrm{~d} \eta^{2}+\sinh ^{2} \eta \mathrm{d} \Omega_{3}^{2}\right), \quad 0 \leq \eta<\infty,
$$


where $d \Omega_{3}$ is the line element on the unit 3-sphere. If we regularize the volume of $\mathrm{AdS}_{4}$ by putting a cut-off $\eta<\eta_{0}$ then the volume is given by

$$
V_{\mathrm{AdS}_{4}}=2 \pi^{2} L^{4}\left(\frac{1}{24} \mathrm{e}^{3 \eta_{0}}-\frac{3}{8} \mathrm{e}^{\eta_{0}}+\frac{2}{3}+\mathcal{O}\left(\mathrm{e}^{-\eta_{0}}\right)\right)
$$

On the other hand the radius of curvature of the boundary 3 -sphere at $\eta=\eta_{0}$ is given by $R=L \sinh \eta_{0}=L\left(\mathrm{e}^{\eta_{0}}-\mathrm{e}^{-\eta_{0}}\right) / 2$. Thus the terms in (3.9) proportional to $\mathrm{e}^{3 \eta_{0}}$ and $\mathrm{e}^{\eta_{0}}$ can be expressed as polynomials in $R$ up to order $1 / R$ corrections and hence can be cancelled by boundary counterterms. As a result we are left with the regularized volume

$$
\operatorname{vol}\left(\mathrm{AdS}_{4}\right)=\frac{4 \pi^{2} L^{4}}{3}
$$

Notice that this is the same regularized value which leads to the successful test of the leading term (1.5). Substituting (3.7) and (3.10) into (2.12) we get

$$
n_{\Delta_{2}}^{0}=1 \text {. }
$$

\subsection{Calculation of $\beta_{2}$}

We now compute $\beta_{2}$. To do this, we proceed as in [16, 17, 18, 19, 20]. The path integral measure over the two-form $B_{\mu \nu}$ in $D$ dimensions is normalized as

$$
\int\left[\mathcal{D} B_{\mu \nu}\right] \exp \left[-\int \mathrm{d}^{D} x \sqrt{g} g^{\mu \nu} g^{\alpha \beta} B_{\mu \alpha} B_{\nu \beta}\right]=1 .
$$

The metric on $\mathrm{AdS}_{4} \times X_{7}$ can be written as $g_{\mu \nu}=L^{2} g_{\mu \nu}^{(0)}$, where $g_{\mu \nu}^{(0)}$ is an $L$-independent metric. The normalization becomes

$$
\int\left[\mathcal{D} B_{\mu \nu}\right] \exp \left[-L^{D-4} \int \mathrm{d}^{D} x \sqrt{g^{(0)}} g^{(0) \mu \nu} g^{(0) \alpha \beta} B_{\mu \alpha} B_{\nu \beta}\right]=1 .
$$

Hence the correctly normalized integration measure corresponds to an integration

$$
\prod_{x,(\mu \nu)} \mathrm{d}\left(L^{D / 2-2} B_{\mu \nu}(x)\right) \text {. }
$$

On the other hand, the zero modes of the $B_{\mu \nu}$ field are associated with the usual gauge transformation of two-forms,

$$
\delta B_{\mu \nu} \propto \partial_{\nu} \theta_{\mu}-\partial_{\mu} \theta_{\nu}
$$

but with non-normalizable $\theta_{\mu}$ so that these are not pure gauge deformations. Now, since we are using a coordinate system in which the metric takes the form $L^{2} g_{\mu \nu}^{(0)}$, the range of coordinates is independent of $L$. Since in any coordinate system we expect $\int \theta_{\mu} \mathrm{d} x^{\mu}$ to have $L$ independent periods, in the coordinate system used here, in which $x^{\mu}$ 's have $L$ independent range, the $\theta_{\mu}$ 's should have $L$ independent integration range. Eq. (3.15) now shows that the integration over each $B_{\mu \nu}$ zero mode has an $L$-independent integration 
range, but due to the $L^{D / 2-2}$ factor in the measure in (3.14) it gives a factor of $L^{D / 2-2}$. Thus we have ${ }^{4}$

$$
\beta_{2}=\frac{D}{2}-2=\frac{7}{2}
$$

for $D=11$.

Since the above result depends crucially on the result for the range of integration of the zero mode of $B_{\mu \nu}$ we shall now elaborate on this further in the context of a compact manifold, regarding the $B_{\mu \nu}$ as the gauge transformation parameters of the 3 -form field. For this let us consider, instead of $\mathrm{AdS}_{4} \times X_{7}$, a compact space with metric $L^{2} g_{\mu \nu}^{(0)}$ where $g_{\mu \nu}^{(0)}$ is $L$ independent. In this case the zero modes of $B_{\mu \nu}$ are harmonic 2-forms, which can be represented locally as $\mathrm{d} \Lambda$ for some one-form $\Lambda$, but this one-form is not globally defined. This is analogous to the 2-form zero modes on $\mathrm{AdS}_{4} \times X_{7}$ which are locally of the form $\mathrm{d} \Lambda$ but the one-form $\Lambda$ is not normalizable. Now returning to the compact case we see that if we regard the two-forms as the gauge transformation parameters of the three-form fields, then the integral of the two form over a 2-cycle of the manifold is a global symmetry transformation parameter, and the corresponding conserved charged is the winding number of the M2-brane on this 2-cycle. Since the latter is quantized in integer units, the integral of the 2 -form over the 2 -cycles will have period $2 \pi$. Since $g_{\mu \nu}^{(0)}$ and hence the coordinate system we have used has no explicit dependence on $L$, this shows that the zero modes of the $B_{\mu \nu}$ fields have $L$ independent integration range.

It is also worth noting that if instead of the 2-form field we had zero modes of the metric - as in the case of [16, 17, 18, 19, 20] - then the result would be different. In this case the metric zero modes would be associated with diffeomorphisms with non-normalizable transformation parameters $\xi^{\mu}(x)$, and the analog of (3.15) would be $\delta g_{\mu \nu}=\nabla_{\mu} \xi_{\nu}+\nabla_{\nu} \xi_{\mu}$. However now the natural variables which have $L$ independent range are the transformation parameters $\xi^{\mu}$, and so when we lower the index with the metric $g_{\mu \nu}$, we get a factor of $L^{2}$ in the range of integration over the metric zero modes [16, 17, 18, 19, 20]. Again the validity of this argument can be checked using the example of a compact manfold. We take the familiar example of a square torus $\mathbb{T}^{2}$ with metric $\mathrm{d} s^{2}=L^{2}\left(\mathrm{~d} x^{2}+\mathrm{d} y^{2}\right)$ and take $x, y$ to have period 1 . Now consider a diffeomorphism $y \rightarrow y+a x, x \rightarrow x$, under which the metric is deformed to $L^{2}\left(\mathrm{~d} x^{2}+(\mathrm{d} y+a \mathrm{~d} x)^{2}\right)$. Since this diffeomorphism does not preserve the periodicity in $x$ and $y$, it is not an allowed diffeomorphism and hence generates a genuine deformation of the metric. Thus this is analogous to non-normalizable diffeomorphisms in the non-compact case. But for $a=1$ the periodicity in $x$ and $y$ is preserved showing that $a=1$ is the same as $a=0$. Hence the parameter $a$ has period 1 , independent of $L$, as we expect on general grounds. Note however that since under this deformation the metric changes by order $L^{2} \delta a$, the range of integration over the metric zero mode is of order $L^{2}$, as predicted from our general arguments.

\subsection{Logarithmic correction to the free energy}

Using (3.5), (3.11) and (3.16) we see that the logarithmic correction to the free energy is

\footnotetext{
${ }^{4}$ Since $B_{\mu \nu}$ are Grassmann variables the factor is actually $L^{-(D / 2-2)}$ but the extra minus sign has already been taken into account in (3.5).
} 
given by

$$
-\left(\beta_{2}-2\right) \log L=-\frac{3}{2} \log L
$$

which precisely matches (1.7).

\section{Conclusions and outlook}

In this note we have shown that the logarithmic correction to the three-sphere partition function, in a large class of three-dimensional CFTs generalizing ABJM theory, can be computed by doing a one-loop calculation in the dual eleven-dimensional supergravity on $\mathrm{AdS}_{4} \times X_{7}$. This can be regarded as a generalization of the program for calculating logarithmic corrections to the black hole entropy developed in [16, 17, 18, 19, 20], and provides a non-trivial test of the AdS/CFT correspondence at next-to-leading order in the $1 / N$ expansion. We have found that this correction is due only to zero modes, more precisely, to the zero mode of a ghost two-form appearing in the quantization of the three-form field of supergravity. This explains its universality: on the field theory side, the correction is independent of the data of the CFT, and on the supergravity side, it is independent of the seven-dimensional manifold $X_{7}$.

The computation we have done here can be extended in various directions. For example, it would be interesting to reproduce the logarithmic shift in the type IIA string picture obtained by dimensional reduction of the eleven-dimensional supergravity backgrounds considered in this paper, which leads to backgrounds of the form $\mathrm{AdS}_{4} \times X_{6}$. In this case there will be contributions from both non-zero and zero-modes, and the answer depends in principle on the details of the six-dimensional manifold $X_{6}$ appearing in the compactification. Another interesting extension concerns the study of logarithmic corrections for type IIA string theory on the backgrounds of the form $\operatorname{AdS}_{6} \times X_{4}$ found in [36]. In this case the partition function can be also computed in the CFT side, and it agrees with the gravity dual at large $N$ [37, so one might try to compare the logarithmic corrections.

\section{Acknowledgements}

We would like to thank Matthias Gaberdiel, Juan Maldacena, Ruben Minasian, Gregory Moore, Binata Panda, Martin Rocek and Arkady Tseytlin for useful conversations. M.M. would like to thank the 2011 Simons Workshop for hospitality in the preliminary stages of this work. The work of A.G. and M.M. is supported by the Fonds National Suisse under subsidies 200020-126817 and 200020-137523. The work of S.B. and A.S. is supported in part by the project 11-R\&D-HRI-5.02-0304. The work of A.S. is also supported in part by the J. C. Bose fellowship of the Department of Science and Technology, India.

\section{References}

[1] O. Aharony, O. Bergman, D. L. Jafferis and J. Maldacena, "N=6 superconformal Chern-Simons-matter theories, M2-branes and their gravity duals," JHEP 0810, 091 (2008) [arXiv:0806.1218 [hep-th]]. 
[2] D. L. Jafferis and A. Tomasiello, "A Simple class of N=3 gauge/gravity duals," JHEP 0810, 101 (2008) [arXiv:0808.0864 [hep-th]].

[3] H. Fuji, S. Hirano, S. Moriyama, "Summing Up All Genus Free Energy of ABJM Matrix Model," JHEP 1108, 001 (2011). [arXiv:1106.4631 [hep-th]].

[4] M. Mariño and P. Putrov, "ABJM theory as a Fermi gas," J. Stat. Mech. 1203, P03001 (2012) [arXiv:1110.4066 [hep-th]].

[5] M. Mariño and P. Putrov, "Exact Results in ABJM Theory from Topological Strings," JHEP 1006, 011 (2010) [arXiv:0912.3074 [hep-th]].

[6] N. Drukker, M. Mariño and P. Putrov, "From weak to strong coupling in ABJM theory," Commun. Math. Phys. 306, 511 (2011) [arXiv:1007.3837 [hep-th]].

[7] C. P. Herzog, I. R. Klebanov, S. S. Pufu and T. Tesileanu, "Multi-Matrix Models and Tri-Sasaki Einstein Spaces," Phys. Rev. D 83, 046001 (2011) [arXiv:1011.5487 [hep-th]].

[8] N. Drukker, M. Mariño and P. Putrov, "Nonperturbative aspects of ABJM theory," JHEP 1111, 141 (2011) [arXiv:1103.4844 [hep-th]].

[9] M. Hanada, M. Honda, Y. Honma, J. Nishimura, S. Shiba and Y. Yoshida, "Numerical studies of the ABJM theory for arbitrary N at arbitrary coupling constant," JHEP 1205, 121 (2012) [arXiv:1202.5300 [hep-th]].

[10] D. R. Gulotta, C. P. Herzog and S. S. Pufu, "From Necklace Quivers to the F-theorem, Operator Counting, and T(U(N))," JHEP 1112, 077 (2011) [arXiv:1105.2817 [hep-th]].

[11] M. Mariño, "Lectures on localization and matrix models in supersymmetric Chern-Simons-matter theories," J. Phys. A A 44, 463001 (2011) [arXiv:1104.0783 [hep-th]].

[12] O. Bergman and S. Hirano, "Anomalous radius shift in AdS(4)/CFT(3)," JHEP 0907, 016 (2009) [arXiv:0902.1743 [hep-th]].

[13] O. Aharony, A. Hashimoto, S. Hirano and P. Ouyang, "D-brane Charges in Gravitational Duals of 2+1 Dimensional Gauge Theories and Duality Cascades," JHEP 1001, 072 (2010) [arXiv:0906.2390 [hep-th]].

[14] A. Castro and S. Murthy, "Corrections to the statistical entropy of five dimensional black holes," JHEP 0906, 024 (2009) [arXiv:0807.0237 [hep-th]].

[15] M. Mariño and P. Putrov, "Interacting fermions and N=2 Chern-Simons-matter theories," arXiv:1206.6346 [hep-th].

[16] S. Banerjee, R. K. Gupta and A. Sen, "Logarithmic Corrections to Extremal Black Hole Entropy from Quantum Entropy Function," JHEP 1103, 147 (2011) [arXiv:1005.3044 [hep-th]].

[17] S. Banerjee, R. K. Gupta, I. Mandal and A. Sen, "Logarithmic Corrections to N=4 and N=8 Black Hole Entropy: A One Loop Test of Quantum Gravity," JHEP 1111, 143 (2011) [arXiv:1106.0080 [hep-th]].

[18] A. Sen, "Logarithmic Corrections to $\mathrm{N}=2$ Black Hole Entropy: An Infrared Window into the Microstates," arXiv:1108.3842 [hep-th]. 
[19] A. Sen, "Logarithmic Corrections to Rotating Extremal Black Hole Entropy in Four and Five Dimensions," Gen. Rel. Grav. 44, 1947 (2012) [arXiv:1109.3706 [hep-th]].

[20] S. Bhattacharyya, B. Panda and A. Sen, "Heat Kernel Expansion and Extremal Kerr-Newmann Black Hole Entropy in Einstein-Maxwell Theory," JHEP 1208, 084 (2012) [arXiv:1204.4061 [hep-th]].

[21] A. Sen, "Logarithmic Corrections to Schwarzschild and Other Non-extremal Black Hole Entropy in Different Dimensions," arXiv:1205.0971 [hep-th].

[22] S. M. Christensen, M. J. Duff, "Quantizing Gravity with a Cosmological Constant," Nucl. Phys. B170, 480 (1980).

[23] R. B. Mann and S. N. Solodukhin, "Universality of quantum entropy for extreme black holes," Nucl. Phys. B 523, 293 (1998) [hep-th/9709064].

[24] M. J. Duff and D. J. Toms, "Kaluza-Klein Kounterterms," in J. Ellis and S. Ferrara (eds), Unification of the Fundamental Particle Interactions II, Plenum, New York, 1983, p. 29.

[25] E. S. Fradkin and A. A. Tseytlin, "Quantum Properties Of Higher Dimensional And Dimensionally Reduced Supersymmetric Theories," Nucl. Phys. B 227, 252 (1983).

[26] D. V. Vassilevich, "Heat kernel expansion: User's manual," Phys. Rept. 388, 279 (2003) [hep-th/0306138].

[27] H. Liu and M. Mezei, "A Refinement of entanglement entropy and the number of degrees of freedom," arXiv:1202.2070 [hep-th].

[28] R. Camporesi and A. Higuchi, "Spectral functions and zeta functions in hyperbolic spaces," J. Math. Phys. 35, 4217 (1994).

[29] R. Camporesi and A. Higuchi, "The Plancherel measure for $p$-forms in real hyperbolic spaces," J. Geom. Phys. 15, 57 (1994).

[30] W. Siegel, "Hidden Ghosts," Phys. Lett. B 93, 170 (1980).

[31] J. Thierry-Mieg, "Brs Structure Of The Antisymmetric Tensor Gauge Theories," Nucl. Phys. B 335, 334 (1990).

[32] E. J. Copeland and D. J. Toms, "Quantized Antisymmetric Tensor Fields And Selfconsistent Dimensional Reduction In Higher Dimensional Space-times," Nucl. Phys. B 255, 201 (1985).

[33] M. S. Volkov and A. Wipf, "Black hole pair creation in de Sitter space: A Complete one loop analysis," Nucl. Phys. B 582, 313 (2000) [hep-th/0003081].

[34] M. J. Duff, B. E. W. Nilsson and C. N. Pope, "Kaluza-Klein Supergravity," Phys. Rept. 130, 1 (1986).

[35] D. E. Diaz and H. Dorn, "Partition functions and double-trace deformations in AdS/CFT," JHEP 0705, 046 (2007) [hep-th/0702163 [HEP-TH]].

[36] O. Bergman and D. Rodriguez-Gomez, "5d quivers and their AdS(6) duals," JHEP 1207, 171 (2012) [arXiv:1206.3503 [hep-th]]. 
[37] D. L. Jafferis and S. S. Pufu, "Exact results for five-dimensional superconformal field theories with gravity duals," arXiv:1207.4359 [hep-th]. 